\title{
On the Adjoint of the Eulerian Idempotent in an Analytic Context
}

\section{Gabriel Pietrzkowski ${ }^{1}$}

Received: 21 February 2018 / Revised: 5 September 2018 / Published online: 20 November 2018

(C) The Author(s) 2018

\begin{abstract}
We generalize Gehrig-Kawski theorem connecting the adjoint of the Eulerian idempotent with the logarithm of identity operator in the convolution product algebra $\operatorname{End}(\mathbb{K}\langle A\rangle)$. This has application in dynamical systems, control theory, coordinates of the first kind, generalized BCH-formula, Magnus expansion, etc., and is connected with iterated integrals and the signature of a path. We also show certain algebraic identities, which are meaningful in context of control and path-signature theory.
\end{abstract}

Keywords Eulerian idempotent $\cdot \mathrm{BCH}$-formula $\cdot$ Signature of a path

Mathematics Subject Classification (2010) 17B01 · 17B80

\section{Introduction}

The Eulerian idempotent $\pi_{1}: \mathbb{K}\langle\mathrm{A}\rangle \rightarrow \mathbb{K}\langle\mathrm{A}\rangle$ can be explicitly defined as a linear endomorphism satisfying

$$
\pi_{1}(w)=\sum_{k=1}^{\infty} \frac{(-1)^{k-1}}{k} \sum_{u_{1}, \ldots, u_{k} \in \mathrm{A}_{+}^{*}}\left(w \mid u_{1} w \cdots \omega u_{k}\right) u_{1} \cdots u_{k}
$$

for all $w \in \mathrm{A}^{*}$. Its importance comes, however, from a more implicate (but natural) definition $\pi_{1}=\log I d$, where the logarithm is taken in the algebra End( $\left.\mathbb{K}\langle A\rangle\right)$ with the convolution product $*$. The name "Eulerian idempotent" emanates from the Eulerian numbers, which are related with coefficients (in symmetric group algebras) of higher order Eulerian idempotents $\pi_{n}=\frac{1}{n !} \pi_{1}^{* n}$ [1]. $\pi_{1}$ is interesting from many points of view. Purely mathematical aspects concern free Lie algebras, symmetric algebras, Solomon algebras, preLie algebras, etc. In this article, we focus our attention on algebraic applications in dynamical systems, control theory, coordinates of the first kind, generalized $\mathrm{BCH}$-formula, Magnus expansion, etc., which are connected with iterated integrals, the signature of a path and so

Gabriel Pietrzkowski

G.Pietrzkowski@mini.pw.edu.pl

1 Faculty of Mathematics and Information Science, Warsaw University of Technology, Warsaw, Poland 
on. In detail, Sussmann [22] showed a product expansion for the solution of a non-linear control-affine system in terms of Lyndon basis of the free Lie algebra on words assigned to the controls. Then Melançon and Reutenauer [16] discovered the same expansion in purely algebraic context, and Reutenauer [20] generalized it to a Hall basis setting. This was rewritten in control-theoretic setting by Kawski and Sussmann [11]. The generalized algebraic version of these results is as follows:

$$
\sum_{w \in \mathrm{A}^{*}} w \otimes w=\prod_{h \in \mathcal{H}} \exp \left(\mathcal{S}_{h} \otimes \mathcal{P}_{h}\right)
$$

In this formula, it is crucial that exp is taken with respect to a product $\operatorname{sh} \otimes$ conc (see Section 2.2 for the definitions), and $\mathcal{H}$ is a Hall set (see Section 2.4 for the definitions of $\left.\mathcal{H}, \mathcal{P}_{h}, \mathcal{S}_{h}\right)$. After a decade, Gehrig [8] and Kawski with Gehrig [7] proved that the adjoint homomorphism $\pi_{1}^{\prime}: \mathbb{K}\langle\langle\mathrm{A}\rangle\rangle \rightarrow \mathbb{K}\langle\langle\mathrm{A}\rangle\rangle$ gives rise to another formula as follows:

$$
\sum_{w \in \mathrm{A}^{*}} w \otimes w=\exp \left(\sum_{h \in \mathcal{H}} \pi_{1}^{\prime}\left(\mathcal{S}_{h}\right) \otimes \mathcal{P}_{h}\right)
$$

with the same data as previously. They work in a context of control theory. Namely, for a control system with the following:

$$
\dot{x}(t)=\sum_{i=1}^{m} u_{i}(t) f_{i}(x(t)) \quad x \in \mathbb{R}^{n},\left(u_{1}, \ldots, u_{m}\right)^{T} \in U \subset \mathbb{R}^{m}
$$

the above formula gives solution as follows:

$$
x(t)=\exp \left(\sum_{h \in \mathcal{H}} \phi^{t} \circ \pi_{1}^{\prime}\left(\mathcal{S}_{h}\right) X_{h}\right)(x(0)),
$$

where $\phi^{t}$ is a linear mapping defined for $a_{1} \cdots a_{k} \in \mathrm{A}_{k}^{*}$ by

$$
\phi^{t}\left(a_{1} \cdots a_{k}\right)=\int_{0}^{t} \cdots \int_{0}^{t_{2}} u_{a_{1}}\left(t_{1}\right) \cdots u_{a_{k}}\left(t_{k}\right) \mathrm{dt}_{1} \cdots \mathrm{dt}_{k}
$$

and $X_{h}$ are appropriate vector fields. In particular, for fixed controls $u_{i}$ and a fixed $t>0$, the solution $x(t)$ is an image of $x(0)$ under the $t$-time flow of a certain vector field. For varying $t$, the vector field is also $t$-varying. This is also connected with the theory of rough paths [12-14]. Namely, for a basis $\left(e_{i}\right)$ of $\mathbb{R}^{m}$, take an alphabet $\mathrm{A}=\left\{e_{i}\right\}$; define a linear isomorphism $\iota_{\otimes}: \mathbb{K}\langle\mathrm{A}\rangle \rightarrow T^{\otimes} \mathbb{R}^{m}$ by $\iota_{\otimes}\left(e_{i_{1}} \cdots e_{i_{k}}\right)=e_{i_{1}} \otimes \cdots \otimes e_{i_{k}}$. The signature of a path $\gamma:[0, T] \rightarrow \mathbb{R}^{m}$ is as follows:

$$
T^{\bar{\otimes}} \mathbb{R}^{m} \ni X(\gamma)=1+\sum_{k=1}^{\infty} \int_{0}^{T} \cdots \int_{0}^{t_{2}} d \gamma_{t_{1}} \otimes \cdots \otimes d \gamma_{t_{k}}
$$

and Gehrig-Kawski formula gives the logarithm of the signature as follows:

$$
\log X(\gamma)=\sum_{h \in \mathcal{H}} \phi_{\gamma} \circ \pi_{1}^{\prime}\left(\mathcal{S}_{h}\right) \iota_{\otimes}\left(\mathcal{P}_{h}\right),
$$

where $\phi_{\gamma}: \mathbb{K}\langle\mathrm{A}\rangle \rightarrow \mathbb{K}$ is a homomorphism defined by $\phi_{\gamma}(P)=\left(X(\gamma) \mid \iota_{\otimes}(P)\right)$.

In this article, we firstly generalize Gehrig-Kawski's result to a non-Hall basis (which means to all basis) of free Lie algebra Lie(A) (this is a more general answer to the Problem 1 stated in [10]). We prove that for a basis $\left\{P_{h} \mid h \in \mathrm{B}\right\}$ in $\operatorname{Lie}(\mathrm{A})$ and a projection 
$\rho: \mathbb{K}\langle\mathrm{A}\rangle \rightarrow \operatorname{Lie}(\mathrm{A}) \subset \mathbb{K}\langle\mathrm{A}\rangle$, there exist the canonical subspace $\operatorname{Lie}_{\rho}^{*}(\mathrm{~A}) \subset \mathbb{K}\langle\langle\mathrm{A}\rangle\rangle$ and its basis $\left\{S_{h} \mid h \in \mathrm{B}\right\}$ for which is as follows:

$$
\sum_{w \in \mathrm{A}^{*}} w \otimes w=\exp \left(\sum_{h \in \mathrm{B}} \pi_{1}^{\prime}\left(S_{h}\right) \otimes P_{h}\right)
$$

We state this result in Theorem 1. After this, we give examples for this theorem choosing different basis in Lie(A) and a projection $\rho$. In particular, we obtain the Gehrig-Kawski formula for a Hall basis.

In the second part of the article, we focus on the aforementioned mappings $\phi^{t} \circ \pi_{1}^{\prime}$ and $\phi_{\gamma} \circ \pi_{1}^{\prime}$ as particular cases of a mapping $\phi \circ \pi_{1}^{\prime}$, with $\phi: \mathbb{K}_{w}\langle\langle\mathrm{~A}\rangle\rangle \rightarrow R$ an algebra homomorphism ( $R$ is a $\mathbb{K}$-algebra). We show in Theorem 3 that for $w \in \mathrm{A}_{m}^{*}$, it follows:

$$
\phi \circ \pi_{1}^{\prime}(w)=\sum_{n=1}^{m} \frac{(-1)^{n+1}}{n}\left(\begin{array}{l}
m \\
n
\end{array}\right) \phi_{n}(w)
$$

where $\phi_{n}(w)=\sum_{w_{1} \cdots w_{k}=w} \phi\left(w_{1}\right) \cdots \phi\left(w_{k}\right)$. From this theorem, we deduce that for a path $\gamma$, the homogeneous components of the logarithmic signature of $\gamma$ can be expressed through the following:

$$
\phi_{\gamma} \circ \pi_{1}^{\prime}(w)=\sum_{n=1}^{m} \frac{(-1)^{n+1}}{n}\left(\begin{array}{c}
m \\
n
\end{array}\right) \phi_{\gamma^{\star n}}(w)
$$

defined for $w \in \mathrm{A}_{m}^{*}$. This formula explicitly connects the $m$-homogeneous component of $\log X(\gamma)$ with the signatures $X\left(\gamma^{\star n}\right)(n=1, \ldots, m)$ of the $n$th powers concatenation of $\gamma$.

\section{Preliminary}

In the article, we assume $\mathbb{N}$ to be the set of natural numbers beginning from $1, \mathbb{K}$ to be a field of characteristic 0 . For a unitary associative $\mathbb{K}$-algebra $(\mathrm{Alg}, \bullet)$ with unit 1 , we use standard notations as follows:

$$
\exp (Q)=\sum_{k=0}^{\infty} \frac{1}{k !} Q^{\bullet k}, \quad \log (1+Q)=\sum_{k=1}^{\infty} \frac{(-1)^{k-1}}{k} Q^{\bullet k}
$$

for any $Q \in \mathrm{Alg}$, for which it makes sense. Here, $Q^{\bullet k}$ is defined recursively by $Q^{\bullet 0}=1$, and $Q^{\bullet k}=Q \bullet Q^{\bullet k-1}$ for $k \in \mathbb{N}$.

\subsection{Tensor Algebra}

Let $V$ be a finite dimensional linear space over $\mathbb{K}$. For $k \in \mathbb{N}$, denote by $V^{\otimes k}$ the $k$ th tensor product of $V ; V^{\otimes 0}=\mathbb{K}$. Let $T^{\otimes} V=\bigoplus_{k=0}^{\infty} V^{\otimes k}$ and $T^{\bar{\otimes}} V$ be its algebraic closure. Consider $\otimes: T^{\bar{\otimes}} V \times T^{\bar{\otimes}} V \rightarrow T^{\bar{\otimes}} V$ as a product, i.e., $V^{\otimes k} \times V^{\otimes l} \ni(x, y) \mapsto x \otimes y \in$ $V^{\otimes k+l}$. Then $\left(T^{\otimes} V, \otimes\right)$ and $\left(T^{\bar{\otimes}} V, \otimes\right)$ are associative $K$-algebras with $1 \in \mathbb{K}=V^{\otimes 0}$ as the unit. 


\subsection{Shuffle Algebra}

Let $A$ be a certain finite set of cardinality $\geq 2$, called the alphabet. Denote by $\mathrm{A}_{n}^{*}, \mathrm{~A}^{*}, \mathbb{K}\langle\mathrm{A}\rangle, \mathbb{K}\langle\langle\mathrm{A}\rangle\rangle$, the set of words of length $n$, the set of all words, the $K$-algebras of non-commutative polynomials, and series in the letters $A$, respectively; by $1 \in \mathrm{A}_{0}^{*}$, we denote the empty word; by $\mathrm{A}_{+}^{*} \subset \mathrm{A}^{*}, \mathbb{K}_{+}\langle\mathrm{A}\rangle \subset \mathbb{K}\langle\mathrm{A}\rangle, \mathbb{K}_{+}\langle\langle\mathrm{A}\rangle\rangle \subset \mathbb{K}\langle\langle\mathrm{A}\rangle\rangle$, the subset of non-trivial (non-empty) words, the submodule spanned on non-trivial words, and its algebraic closure, respectively. The module $\mathbb{K}\langle\langle\mathrm{A}\rangle\rangle$ is dual to $\mathbb{K}\langle\mathrm{A}\rangle$, and we identify $P \in \mathbb{K}\langle\langle\mathrm{A}\rangle\rangle$ with the $\mathbb{K}$-linear functional $(P \mid \cdot): \mathbb{K}\langle\mathrm{A}\rangle \rightarrow \mathbb{K}$ by writing $P=\sum_{w \in \mathrm{A}^{*}}(P \mid w) w$. The product of two series $P, Q \in \mathbb{K}\langle\langle\mathrm{A}\rangle\rangle$ is therefore defined by $(\mathrm{PQ} \mid w):=\sum_{\mathrm{uv}=w}(P \mid u)(Q \mid v)$. Since $\mathbb{K}\langle\mathrm{A}\rangle$ is a finite-free generated algebra, the module $\mathbb{K}\langle\mathrm{A}\rangle$ is dual to $\mathbb{K}\langle\langle\mathrm{A}\rangle\rangle$, and we also identify $Q \in \mathbb{K}\langle\mathrm{A}\rangle$ with $(Q \mid \cdot): \mathbb{K}\langle\langle\mathrm{A}\rangle\rangle \rightarrow \mathbb{K}$ by $Q=\sum_{w \in \mathrm{A}^{*}}(Q \mid w) w$. Clearly, $(P \mid Q)=(Q \mid P)$, and it also plays a role of a scalar product in $\mathbb{K}\langle\mathrm{A}\rangle$. For a $\mathbb{K}$-submodule $Y \subset \mathbb{K}\langle\mathrm{A}\rangle$, we denote by $Y^{\perp}=\{S \in \mathbb{K}\langle\langle\mathrm{A}\rangle\rangle \mid(S \mid y)=0 \forall y \in Y\} \subset \mathbb{K}\langle\langle\mathrm{A}\rangle\rangle$ the module of series representing functionals vanishing on $Y$. If $X, Y \subset \mathbb{K}\langle\mathrm{A}\rangle$ are $\mathbb{K}$-submodules then for an endomorphism $\rho: X \rightarrow Y$, we denote $\operatorname{ker}^{\perp} \rho:=(\operatorname{ker} \rho)^{\perp}$.

Since $\mathbb{K}\langle A\rangle \subset \mathbb{K}\langle\langle\mathrm{A}\rangle\rangle$, and $\mathbb{K}\langle\langle\mathrm{A}\rangle\rangle$ is the algebraic closure of $\mathbb{K}\langle\mathrm{A}\rangle$, we define all objects in the larger algebra. The concatenation product in $\mathbb{K}\langle\langle\mathrm{A}\rangle\rangle$ has its tensorial version which we denote by conc $: \mathbb{K}\langle\langle\mathrm{A}\rangle\rangle \otimes \mathbb{K}\langle\langle\mathrm{A}\rangle\rangle \rightarrow \mathbb{K}\langle\langle\mathrm{A}\rangle\rangle$, i.e., $\operatorname{conc}(P \otimes Q)=\mathrm{PQ}$. Introduce another-shuffle-product $w: \mathbb{K}\langle\langle\mathrm{A}\rangle\rangle \otimes \mathbb{K}\langle\langle\mathrm{A}\rangle\rangle \rightarrow \mathbb{K}\langle\langle\mathrm{A}\rangle\rangle$ defined recursively for words by $1 \omega w=w ш 1=w$ for any $w \in \mathrm{A}^{*}$, and

$$
\left(w_{1} a_{1}\right) ш\left(w_{2} a_{2}\right)=\left(w_{1} ш\left(w_{2} a_{2}\right)\right) a_{1}+\left(\left(w_{1} a_{1}\right) ш w_{2}\right) a_{2}
$$

for all $a_{1}, a_{2} \in \mathrm{A}$ and $w_{1}, w_{2} \in \mathrm{A}^{*}$. In what follows, we will use another standard notation for the shuffle product $\operatorname{sh}: \mathbb{K}\langle\langle\mathrm{A}\rangle\rangle \otimes \mathbb{K}\langle\langle\mathrm{A}\rangle\rangle \rightarrow \mathbb{K}\langle\langle\mathrm{A}\rangle\rangle$, i.e., $\operatorname{sh}\left(w_{1} \otimes w_{2}\right)=w_{1} w w_{2}$, and its generalized version $\operatorname{sh}_{k}: \mathbb{K}\langle\langle\mathrm{A}\rangle\rangle^{\otimes k} \rightarrow \mathbb{K}\langle\langle\mathrm{A}\rangle\rangle$ given by the following:

$$
\operatorname{sh}_{k}\left(w_{1} \otimes \cdots \otimes w_{k}\right):=w_{1} ш \ldots ш w_{k}
$$

for $w_{1}, \ldots, w_{k} \in \mathrm{A}^{*}$. We denote by $\mathbb{K}_{w}\langle\mathrm{~A}\rangle\left(\right.$ resp. $\left.\mathbb{K}_{u}\langle\langle\mathrm{~A}\rangle\rangle\right)$ the commutative $\mathbb{K}$-algebras of non-commutative polynomials (resp. series) in the letters A with $w$ as the product. The adjoint coproduct of the shuffle product $\delta=\delta_{2}: \mathbb{K}\langle\langle\mathrm{A}\rangle\rangle \rightarrow \mathbb{K}\langle\langle\mathrm{A}\rangle\rangle \otimes \mathbb{K}\langle\langle\mathrm{A}\rangle\rangle$ and its generalized version $\delta_{k}: \mathbb{K}\langle\langle\mathrm{A}\rangle\rangle \rightarrow \mathbb{K}\langle\langle\mathrm{A}\rangle\rangle^{\otimes k}$ are defined by the following:

$$
\delta_{k}(w):=\sum_{w_{1}, \ldots, w_{k} \in \mathrm{A}^{*}}\left(w \mid w_{1} w \cdots ш w_{k}\right) w_{1} \otimes \cdots \otimes w_{k} .
$$

Similarly, we define the adjoint coproduct of the concatenation $\delta^{\prime}=\delta_{2}^{\prime}: \mathbb{K}\langle\langle\mathrm{A}\rangle\rangle \rightarrow \mathbb{K}\langle\langle\mathrm{A}\rangle\rangle \otimes \mathbb{K}\langle\langle\mathrm{A}\rangle\rangle$ and its generalized version $\delta_{k}^{\prime}: \mathbb{K}\langle\langle\mathrm{A}\rangle\rangle \rightarrow \mathbb{K}\langle\langle\mathrm{A}\rangle\rangle^{\otimes k}$ by the following;

$$
\delta_{k}^{\prime}(w):=\sum_{w_{1} \cdots w_{k}=w} w_{1} \otimes \cdots \otimes w_{k},
$$

where the sum is taken over all $w_{i} \in \mathrm{A}^{*}$. Note that $(\mathbb{K}\langle\mathrm{A}\rangle$, conc, $\delta)$ and $\left(\mathbb{K}\langle\langle\mathrm{A}\rangle\rangle\right.$, sh, $\left.\delta^{\prime}\right)$ are mutually adjoint bialgebras [20, Prop. 1.9].

\subsection{Free Lie Algebra on A}

Let $[\cdot, \cdot]: \mathbb{K}\langle\mathrm{A}\rangle \times \mathbb{K}\langle\mathrm{A}\rangle \rightarrow \mathbb{K}\langle\mathrm{A}\rangle$ be the standard Lie bracket, i.e., a bilinear mapping given by $[P, Q]:=\mathrm{PQ}-\mathrm{QP}$. We denote by Lie(A) the smallest $\mathbb{K}$-submodule of $\mathbb{K}\langle\mathrm{A}\rangle$, which 
contains A, and is closed under the Lie bracket, i.e., the free Lie algebra generated by A, and by $\operatorname{Lie}((\mathrm{A}))$ its algebraic closure. By Lie*(A), we denote the module of $\mathbb{K}$-linear functionals $\operatorname{Lie}(\mathrm{A}) \rightarrow \mathbb{K}$. Denote by $(S\lfloor X)$ the action of a $\mathbb{K}$-homomorphism $S: \operatorname{Lie}(\mathrm{A}) \rightarrow \mathbb{K}$ on an element $X \in \operatorname{Lie}(\mathrm{A})$. Let B be an totally ordered set, and $\left\{P_{h} \mid h \in \mathrm{B}\right\}$ be a basis in Lie(A). Let $\left\{S_{h} \mid h \in \mathrm{B}\right\}$ be the dual basis of $\operatorname{Lie}^{*}(\mathrm{~A})$, i.e., the one defined by the following:

$$
X=\sum_{h \in \mathrm{B}}\left(S_{h}\lfloor X) P_{h}\right.
$$

for any $X \in \operatorname{Lie}(\mathrm{A})$.

\subsection{Hall Sets \& Basis}

Let $\mathcal{M}(\mathrm{A})$ be the set of binary, complete, planar, rooted trees with leaves labeled by $\mathrm{A}$. Each such tree can be naturally identified with the unique expression in the set $\mathcal{E}(\mathrm{A})$ defined by the following two conditions: (i) if $a \in \mathrm{A}$, then $a \in \mathcal{E}(\mathrm{A})$, and (ii) if $t, t^{\prime} \in \mathcal{E}(\mathrm{A})$, then $\left(t, t^{\prime}\right) \in \mathcal{E}(\mathrm{A})$. In the sequel, we will not distinguish between these sets, i.e., we assume $\mathcal{M}(\mathrm{A})=\mathcal{E}(\mathrm{A})$. Define the mapping $\mathcal{F}(\cdot): \mathcal{M}(\mathrm{A}) \rightarrow \mathrm{A}^{*}$, which assigns to a tree $t \in \mathcal{M}(\mathrm{A})$, the word given by dropping all brackets in it, i.e., $\mathcal{F}(a)=a$ for all $a \in \mathrm{A}$, and $\mathcal{F}\left(\left(t, t^{\prime}\right)\right)=\mathcal{F}(t) \mathcal{F}\left(t^{\prime}\right)$ for all $t, t^{\prime} \in \mathcal{M}(\mathrm{A})$. The word $\mathcal{F}(t)$ is called the foliage of $t \in \mathcal{M}(\mathrm{A})$. Define also the mapping $\mathcal{P}$. $: \mathcal{M}(\mathrm{A}) \rightarrow \operatorname{Lie}(\mathrm{A})$, which changes the rounded brackets into the Lie brackets, i.e., $\mathcal{P}_{a}=a$ for all $a \in \mathrm{A}$, and $\mathcal{P}_{\left(t, t^{\prime}\right)}:=\left[\mathcal{P}_{t}, \mathcal{P}_{t^{\prime}}\right]$ for all $t, t^{\prime} \in \mathcal{M}(\mathrm{A})$. We will generalize this definition in the sequel. A Hall set $\mathcal{H}$ on the letters $\mathrm{A}$ $[9]^{1}$, is a subset of $\mathcal{M}(\mathrm{A})$ totally ordered by $\leq$ and satisfying:

(I) $\mathrm{A} \subset \mathcal{H}$;

(II) if $h=\left(h^{\prime}, h^{\prime \prime}\right) \in \mathcal{H} \backslash \mathrm{A}$, then $h^{\prime \prime} \in \mathcal{H}$ and $h<h^{\prime \prime}$;

(III) for all $h=\left(h^{\prime}, h^{\prime \prime}\right) \in \mathcal{M}(\mathrm{A}) \backslash$ A we have $h \in \mathcal{H}$ iff

- $\quad h^{\prime}, h^{\prime \prime} \in \mathcal{H}$ and $h^{\prime}<h^{\prime \prime}$, and

- $h^{\prime} \in \mathrm{A}$ or $h^{\prime}=(x, y)$ such that $y \geq h^{\prime \prime}$.

Fix a Hall set $\mathcal{H}$ on the letters A totally ordered by $\leq$. Each Hall tree $h \in \mathcal{H}$ corresponds to a word $\mathcal{F}(h) \in \mathrm{A}^{*}$ called a Hall word. Denote by $\mathcal{W}$, the set of Hall words with ordering $\leq$ inherited from the ordering on $\mathcal{H}$ in the natural way. Each word $w \in \mathrm{A}^{*}$, is the unique concatenation of a unique non-increasing series of Hall words, that is, $w=h_{1} \cdots h_{k}$ for some unique $k \in \mathbb{N}$, and $h_{i} \in \mathcal{W}$ such that $h_{1} \geq \cdots \geq h_{k}$. Let $\mathcal{P}$. : $\mathrm{A}^{*} \rightarrow \mathbb{K}\langle\mathrm{A}\rangle$ be the mapping defined by the following

(i) $\mathcal{P}_{1}:=1$;

(ii) $\mathcal{P}_{a}:=a$ for $a \in \mathrm{A}$;

(iii) $\mathcal{P}_{h}:=\mathcal{P}_{t} \in \operatorname{Lie}(\mathrm{A})$ for $h \in \mathcal{W}$ such that $h=\mathcal{F}(t), t \in \mathcal{H} \subset \mathcal{M}(\mathrm{A})$;

(iv) $\mathcal{P}_{w}:=\mathcal{P}_{h_{1}} \cdots \mathcal{P}_{h_{k}} \in \mathbb{K}\langle\mathrm{A}\rangle$ for $w=h_{1} \cdots h_{k}$, where $k \in \mathbb{N}$ and $h_{i} \in \mathcal{W}$ such that $h_{1} \geq \cdots \geq h_{k}$.

The set $\left\{\mathcal{P}_{h} \in \operatorname{Lie}(\mathrm{A}) \mid h \in \mathcal{W}\right\}$ is the Hall basis of Lie(A) corresponding to the Hall set $\mathcal{H}$. By the Poincaré-Birkhoff-Witt theorem, the set of ordered products $\mathcal{P}_{h_{1}} \cdots \mathcal{P}_{h_{k}}$, where $h_{1} \geq \cdots \geq h_{k}$ are Hall words, creates a basis for the enveloping algebra of Lie(A), which

\footnotetext{
${ }^{1}$ A Hall set should probably be called a Hall-Shirshov-Viennot set because of an important contribution of the other two authors to this theory $[21,23]$.
} 
in the free case is isomorphic to $\mathbb{K}_{+}\langle\mathrm{A}\rangle$. Therefore, $\left\{\mathcal{P}_{w} \mid w \in \mathrm{A}^{*}\right\}$ is a basis in $\mathbb{K}\langle\mathrm{A}\rangle$. Consider the dual basis $\left\{\mathcal{S}_{w} \mid w \in \mathrm{A}^{*}\right\}$ in $\mathbb{K}\langle\langle\mathrm{A}\rangle\rangle$.

Proposition 1 ([20, Theorem 5.3]) (i) $\mathcal{S}_{1}=1$;

(ii) If $h=a v \in \mathcal{W}$ is a Hall word, where a $\in \mathrm{A}, v \in \mathrm{A}^{*}$, then $\mathcal{S}_{h}=a \mathcal{S}_{v}$;

(iii) If $w=h_{1}^{i_{1}} \cdots h_{k}^{i_{k}} \in \mathrm{A}^{*}$ is any word, where $h_{1}>\cdots>h_{k}$ are Hall words and $i_{1}, \ldots, i_{k} \in \mathbb{N}$, then

$$
S_{w}=\frac{1}{i_{1} ! \cdots i_{k} !} S_{h_{1}}^{\uplus i_{1}} \amalg \cdots ш S_{h_{k}}^{ш i_{k}} .
$$

One of the consequences of this proposition is that $\mathbb{K}_{w}\langle\langle\mathrm{~A}\rangle\rangle$ is the algebraic closure of the free commutative algebra over $\left\{\mathcal{S}_{h} \mid h \in \mathcal{W}\right\}$. In particular, $\mathcal{S}_{h_{1}} w \cdots ш \mathcal{S}_{h_{k}}$, where $h_{1} \geq \cdots \geq h_{k}$ are Hall words, creates a basis for $\mathbb{K}_{+}\langle\langle\mathrm{A}\rangle\rangle$.

\subsection{Algebra of Endomorphisms End( $\mathbb{K}\langle A\rangle)$}

Consider $\operatorname{End}(\mathbb{K}\langle\mathrm{A}\rangle)$ - the $\mathbb{K}$-module of linear endomorphisms from $\mathbb{K}\langle\mathrm{A}\rangle$ to $\mathbb{K}\langle\mathrm{A}\rangle$. For $f, g \in \operatorname{End}(\mathbb{K}\langle\mathrm{A}\rangle)$ define their convolution product as follows:

$$
f * g=\operatorname{conc} \circ(f \otimes g) \circ \delta \in \operatorname{End}(\mathbb{K}\langle\mathrm{A}\rangle) .
$$

Let $\epsilon: \mathbb{K}\langle\mathrm{A}\rangle \rightarrow \mathbb{K}\langle\mathrm{A}\rangle$ be a projection $\epsilon(Q)=(Q \mid 1) 1$. Then, $(\operatorname{End}(\mathbb{K}\langle\mathrm{A}\rangle), *)$ is an associative $\mathbb{K}$-algebra with unit $\epsilon$. Introduce the complete tensor product as follows:

$$
\mathcal{A}=\mathbb{K}\langle\langle\mathrm{A}\rangle\rangle \bar{\otimes} \mathbb{K}\langle\mathrm{A}\rangle
$$

and a product $\bar{*}=\operatorname{sh} \otimes$ conc $: \mathcal{A} \otimes \mathcal{A} \rightarrow \mathcal{A}$, i.e.,

$$
\left(P_{1} \otimes Q_{1}\right) \bar{*}\left(P_{2} \otimes Q_{2}\right)=\operatorname{sh}\left(P_{1} \otimes P_{2}\right) \otimes \operatorname{conc}\left(Q_{1} \otimes Q_{2}\right),
$$

for $P_{1}, P_{2} \in \mathbb{K}\langle\langle\mathrm{A}\rangle\rangle, Q_{2}, Q_{2} \in \mathbb{K}\langle\mathrm{A}\rangle$. Then, $(\mathcal{A}, \bar{*})$ is an associative $\mathbb{K}$-algebra with unit $\overline{1}=1 \otimes 1$. The canonical isomorphism of modules $\operatorname{Im}: \operatorname{End}(\mathbb{K}\langle\mathrm{A}\rangle) \rightarrow \mathcal{A}$ given by the following:

$$
\operatorname{Im}(f)=\sum_{u \in \mathrm{A}^{*}} u \otimes f(u)
$$

is a homomorphism of algebras $(\operatorname{End}(\mathbb{K}\langle\mathrm{A}\rangle), *)$ and $(\mathcal{A}, \bar{*})$, i.e.,

$$
\sum_{u \in \mathrm{A}^{*}} u \otimes f * g(u)=\left(\sum_{u \in \mathrm{A}^{*}} u \otimes f(u)\right) \bar{*}\left(\sum_{u \in \mathrm{A}^{*}} u \otimes g(u)\right) .
$$

Note that in the definition of $\operatorname{Im}$, we choose the most natural basis $\left\{u \mid u \in \mathrm{A}^{*}\right\}$ in $\mathbb{K}\langle\mathrm{A}\rangle$ and its dual basis $\left\{u \mid u \in \mathrm{A}^{*}\right\}$ in $\mathbb{K}\langle\langle\mathrm{A}\rangle\rangle$, but in general one can take a different basis and its dual. 


\section{Eulerian Idempotent and its Adjoint}

Let $I: \mathbb{K}\langle\mathrm{A}\rangle \rightarrow \mathbb{K}\langle\mathrm{A}\rangle$ be a projection given by $I(Q)=Q-\epsilon(Q)$. We are particularly interested in an endomorphism $\pi_{1}: \mathbb{K}\langle\mathrm{A}\rangle \rightarrow \mathbb{K}\langle\mathrm{A}\rangle$,

$$
\pi_{1}=\log \mathrm{Id}=\log (\epsilon+I)=\sum_{k=1}^{\infty} \frac{(-1)^{k-1}}{k} I^{* k} .
$$

called the Eulerian idempotent. A straightforward calculation shows that $\operatorname{Im}\left(\pi_{1}\right)$ equals

$$
\sum_{w \in \mathrm{A}^{*}} w \otimes \pi_{1}(w)=\log \left(\sum_{w \in \mathrm{A}^{*}} w \otimes w\right) .
$$

It is known that $\pi_{1}$ is a projection, $\pi_{1}(\mathbb{K}\langle\mathrm{A}\rangle)=\operatorname{Lie}(\mathrm{A})$, and $\left(P \uplus Q \mid \pi_{1}(R)\right)=0$ for all $P, Q, R \in \mathbb{K}_{+}\langle\mathrm{A}\rangle$. The second statement means $\operatorname{ker}^{\perp} \pi_{1}=\operatorname{span}\left\{v w w \mid v, w \in \mathrm{A}_{+}^{*}\right\}$. From Proposition 1, we conclude that

$$
\left\{S_{h_{1}} \uplus \cdots ш S_{h_{k}} \mid k \geq 2, h_{1} \geq \cdots \geq h_{k} \in \mathcal{W}\right\}
$$

is a basis of this space. The kernel of $\pi_{1}$ have another characterization: ker $\pi_{1}=\bigcup_{k \geq 2} \pi_{1}^{* k}(\mathbb{K}\langle\mathrm{A}\rangle)$, where $\pi_{1}^{* k}(\mathbb{K}\langle\mathrm{A}\rangle)=\left\{P^{k} \mid P \in \operatorname{Lie}(\mathrm{A})\right\}$ [20, Thm. 3.7].

The main results in this article concern $\pi_{1}^{\prime}: \mathbb{K}\langle\langle\mathrm{A}\rangle\rangle \rightarrow \mathbb{K}\langle\langle\mathrm{A}\rangle\rangle$ - the adjoint endomorphism to $\pi_{1}$, i.e., the one defined by the following;

$$
\left(\pi_{1}^{\prime}(P) \mid Q\right)=\left(P \mid \pi_{1}(Q)\right)
$$

for all $P \in \mathbb{K}\langle\langle\mathrm{A}\rangle\rangle, Q \in \mathbb{K}\langle\mathrm{A}\rangle$. A straightforward calculation using adjointness of conc $_{k}$ and $\delta_{k}^{\prime}$, adjointness of $\operatorname{sh}_{k}$ and $\delta_{k}$, and self-adjointness of $I$, brings to the following formula

$$
\pi_{1}^{\prime}=\sum_{k \geq 1} \frac{(-1)^{k+1}}{k} \operatorname{sh}_{k} \circ I^{\otimes k} \circ \delta_{k}^{\prime} .
$$

Since $\pi_{1}$ is a projection, the same is true for $\pi_{1}^{\prime}$. In particular, $\pi_{1}^{\prime}(\mathbb{K}\langle\langle\mathrm{A}\rangle\rangle)=\operatorname{ker}^{\perp} \pi_{1}$ and $\operatorname{ker} \pi_{1}^{\prime}=\pi_{1}(\mathbb{K}\langle\mathrm{A}\rangle)^{\perp}=(\operatorname{Lie}(\mathrm{A}))^{\perp}$, which we denote by $\operatorname{Lie}^{\perp}(\mathrm{A})$.

Since $\pi_{1}$ is onto Lie(A), we also consider its adjoint as an epimorphism. Namely, for $\bar{\pi}_{1}: \mathbb{K}\langle\mathrm{A}\rangle \rightarrow \operatorname{Lie}(\mathrm{A}), \bar{\pi}_{1}(P):=\pi_{1}(P)$, we introduce its adjoint endomorphism $\bar{\pi}_{1}^{\prime}: \operatorname{Lie}^{*}(\mathrm{~A}) \rightarrow \mathbb{K}\langle\langle\mathrm{A}\rangle\rangle$. It is a $\mathbb{K}$-linear monomorphism onto $\mathrm{ker}^{\perp} \pi_{1}$ satisfying the following:

$$
\left(\alpha\left\lfloor\pi_{1}(Q)\right)=\left(\bar{\pi}_{1}^{\prime}(\alpha) \mid Q\right)\right.
$$

for all $\alpha \in \mathrm{Lie}^{*}(\mathrm{~A}), Q \in \mathbb{K}\langle\mathrm{A}\rangle$.

Proposition 2 Let $\left\{P_{h} \mid h \in \mathrm{B}\right\}$ and $\left\{S_{h} \mid h \in \mathrm{B}\right\}$ be bases in $\operatorname{Lie}(\mathrm{A})$ and its dual in $\mathrm{Lie}^{*}(\mathrm{~A})$, respectively. It follows that

$$
\mathcal{A} \ni \operatorname{Im}(\mathrm{Id})=\exp \left(\sum_{h \in \mathrm{B}} \bar{\pi}_{1}^{\prime}\left(S_{h}\right) \otimes P_{h}\right) .
$$

Proof We prove that

$$
\log \left(\sum_{w \in \mathrm{A}^{*}} w \otimes w\right)=\sum_{h \in \mathrm{B}} \bar{\pi}_{1}^{\prime}\left(S_{h}\right) \otimes P_{h},
$$


Since $\pi_{1}(\mathbb{K}\langle\mathrm{A} \angle) \subset \operatorname{Lie}(A)$ it follows that

$$
\begin{aligned}
\log \left(\sum_{w \in \mathrm{A}^{*}} w \otimes w\right) & =\sum_{w \in \mathrm{A}^{*}} w \otimes \pi_{1}(w)=\sum_{w \in \mathrm{A}^{*}} w \otimes \sum_{h \in \mathrm{B}}\left(S_{h}\left\lfloor\pi_{1}(w)\right) P_{h}\right. \\
& =\sum_{w \in \mathrm{A}^{*}} w \otimes \sum_{h \in \mathrm{B}}\left(\bar{\pi}_{1}^{\prime}\left(S_{h}\right) \mid w\right) P_{h}=\sum_{h \in \mathrm{B}}\left(\sum_{w \in \mathrm{A}^{*}}\left(\bar{\pi}_{1}^{\prime}\left(S_{h}\right) \mid w\right) w\right) \otimes P_{h} \\
& =\sum_{h \in \mathrm{A}^{*}} \bar{\pi}_{1}^{\prime}\left(S_{h}\right) \otimes P_{h} .
\end{aligned}
$$

This ends the proof.

Choose a basis $\left\{P_{h} \mid h \in \mathrm{B}\right\}$ in $\operatorname{Lie}(\mathrm{A})$, and a projection $\rho: \mathbb{K}\langle\mathrm{A}\rangle \rightarrow \mathbb{K}\langle\mathrm{A}\rangle$ on $\operatorname{Lie}(\mathrm{A})$. Then, $\operatorname{Lie}^{*}(\mathrm{~A})$ is naturally identified with $\operatorname{Lie}_{\rho}^{*}(\mathrm{~A}):=\operatorname{ker}^{\perp} \rho \subset \mathbb{K}\langle\langle\mathrm{A}\rangle\rangle$ by the isomorphism $\iota_{\rho}: \operatorname{Lie}_{\rho}^{*}(\mathrm{~A}) \rightarrow \operatorname{Lie}^{*}(\mathrm{~A})$ given by $\left(\iota_{\rho}(S)\lfloor P)=(S \mid P)\right.$. In $\operatorname{Lie}_{\rho}^{*}(\mathrm{~A})$, there exists the dual basis $\left\{S_{h} \mid h \in \mathrm{B}\right\}$ to the one in $\operatorname{Lie}(\mathrm{A})$ given by the following:

$$
\begin{aligned}
\left(S_{h} \mid Q\right) & =\left(S_{h} \mid \rho(Q)\right) & \forall_{Q \in K\langle\mathrm{~A}\rangle}, \\
P & =\sum_{h \in \mathrm{B}}\left(S_{h} \mid P\right) P_{h} & \forall_{P \in \operatorname{Lie}(\mathrm{A})} .
\end{aligned}
$$

Theorem 1 Let $\left\{P_{h} \mid h \in \mathrm{B}\right\}$ a basis in Lie(A), $\rho: \mathbb{K}\langle\mathrm{A}\rangle \rightarrow \mathbb{K}\langle\mathrm{A}\rangle$ a projection on Lie(A), and $\left\{S_{h} \mid h \in \mathrm{B}\right\}$ the basis in $\mathrm{Lie}_{\rho}^{*}(\mathrm{~A})$ given by (4). It follows that

$$
\mathcal{A} \ni \operatorname{Im}(\mathrm{Id})=\exp \left(\sum_{h \in \mathrm{B}} \pi_{1}^{\prime}\left(S_{h}\right) \otimes P_{h}\right) .
$$

Proof Using Proposition 2 for $\left\{P_{h} \mid h \in \mathrm{B}\right\}$ and $\left\{\iota_{\rho}\left(S_{h}\right) \mid h \in \mathrm{B}\right\}$ we get

$$
\operatorname{Im}(\mathrm{Id})=\exp \left(\sum_{h \in \mathrm{B}} \bar{\pi}_{1}^{\prime}\left(\iota_{\rho}\left(S_{h}\right)\right) \otimes P_{h}\right) .
$$

Since $\left(\iota_{\rho}(\alpha)\left\lfloor\pi_{1}(Q)\right)=\left(\alpha \mid \pi_{1}(Q)\right)\right.$ for all $\alpha \in \operatorname{Lie}_{\rho}^{*}(\mathrm{~A}), Q \in \mathbb{K}\langle\mathrm{A}\rangle$, we have $\bar{\pi}_{1}^{\prime} \circ \iota_{\rho}=\pi_{1}^{\prime}$ on $\operatorname{Lie}_{\rho}^{*}(\mathrm{~A})$. This ends the proof.

A few remarks are in order. First of all, we recall that in the algebra $\mathcal{A}$ the product is $s h \otimes$ conc, so the shuffle product is used to compute the left side of the tensor product. It means that good properties of $\pi_{1}^{\prime}\left(S_{h}\right)$ with respect to sh are welcome. Secondly, the proved formula is similar to the quite clear formula (6.2.1) in Reutenauer's book [20] (in which the sum is taken over all words). In our case, however, the sum is taken over the basis of the Lie algebra, which contains essential information about the logarithm of a series $[4,18]$. Therefore, this theorem generalizes the Gehrig-Kawski theorem (see Theorem 2 beneath) to its most extent. The advantage of the theorem is that it can be used to compute $\mathrm{BCH}$-formula, Magnus expansion, logarithm of the signature, coordinates of the second kind, etc., both in general case, as well as for particular situations. In each 
case, one can try to choose a basis in $\operatorname{Lie}(\mathrm{A})$ and $\rho$ to utilize specific features of a given problem.

There are several natural choices for $\rho$. The first one is $\rho=\pi_{1}$. Recall that $\operatorname{ker} \pi_{1}=\left\{P^{k} \mid k \geq 2, P \in \operatorname{Lie}(\mathrm{A})\right\} \oplus \mathbb{K} 1$ and $\operatorname{Lie}_{\pi_{1}}^{*}(\mathrm{~A})=\operatorname{ker}^{\perp} \pi_{1}=\operatorname{span}\left\{v \omega w \mid v, w \in \mathrm{A}_{+}^{*}\right\}$ in which there are natural basis

$$
\left\{S_{h_{1}} ш \cdots ш S_{h_{k}} \mid k \geq 2, h_{1} \geq \cdots \geq h_{k} \in \mathcal{W}\right\}
$$

written in terms of the dual elements to a Hall basis in Lie(A) [17, Th. 3.1.1]. The description of its dual in $\operatorname{Lie}(\mathrm{A})$ is unknown.

The second one is to take $\rho=\rho_{\perp}$ as the orthogonal projection with respect to the scalar product $(\cdot \mid \cdot)$ in $\mathbb{K}\langle\mathrm{A}\rangle$ (for more details about this projection see [5]). In this case, $\operatorname{Lie}_{\rho_{\perp}}^{*}(\mathrm{~A})=\operatorname{ker}^{\perp} \rho_{\perp}=\operatorname{Lie}((\mathrm{A}))$. In particular, $\rho_{\perp} \neq \pi_{1}$. Take a basis $\left\{P_{h} \mid h \in \mathrm{B}\right\}$ in Lie(A) on a well-ordered set B. Using Gram-Schmidt process obtain the orthonormal basis $\left\{\hat{P}_{h} \mid h \in \mathrm{B}\right\}$. Then, this is also the dual basis in Lie(A). Therefore, from the above theorem, we get $\operatorname{Im}(\mathrm{Id})=\exp \left(\sum_{h \in \mathrm{B}} \pi_{1}^{\prime}\left(\hat{P}_{h}\right) \otimes \hat{P}_{h}\right)$. The same is clearly true for any orthonormal basis in $\operatorname{Lie}(\mathrm{A})$.

The third one is to take $\rho=\rho_{\mathrm{PBW}}$ as the projection with kernel derived from PoincareBirkhoff-Witt theorem. More precisely, universal enveloping algebra of the free Lie algebra Lie(A) is $\mathbb{K}\langle\mathrm{A}\rangle$. If $\left\{P_{h} \mid h \in \mathrm{B}\right\}$ is a basis in Lie(A) then, using Poincaré-Birkhoff-Witt theorem, the set of ordered products $P_{h_{1}} \cdots P_{h_{k}}$, where $h_{1} \geq \cdots \geq h_{k} \in \mathrm{B}$, is a basis for the enveloping algebra of $\operatorname{Lie}(\mathrm{A})$, i.e., in $\mathbb{K}_{+}\langle\mathrm{A}\rangle$. Denote by $S_{h_{1}, \ldots, h_{k}}, h_{1} \geq \cdots \geq h_{k} \in$ $\mathrm{B}$, the elements of the dual basis in $\mathbb{K}_{+}\langle\langle\mathrm{A}\rangle\rangle$. We define $\rho_{\mathrm{PBW}}$ as a projection on Lie(A) with the kernel $\operatorname{ker} \rho_{\mathrm{PBW}}=\operatorname{span}\left\{P_{h_{1}} \cdots P_{h_{k}} \mid k \geq 2, h_{1} \geq \cdots \geq h_{k} \in \mathrm{B}\right\} \oplus \mathbb{K} 1$. From PBW theorem, the elements written in this formula are linearly independent, hence creates a basis of ker $\rho_{\mathrm{PBW}}$. On the dual side, $\operatorname{ker}^{\perp} \rho_{\mathrm{PBW}}=\operatorname{span}\left\{S_{h} \mid h \in \mathrm{B}\right\}$, but in general case, there is not known explicit formulas for $S_{h}$. It follows that ker $\rho_{\mathrm{PBW}} \neq \operatorname{ker} \pi_{1}$, because for $h>h^{\prime} \in$ B, we have ker $\pi_{1} \ni\left(P_{h}+P_{h^{\prime}}\right)^{2}=P_{h}^{2}+P_{h^{\prime}}^{2}+2 P_{h} P_{h^{\prime}}-\left[P_{h}, P_{h^{\prime}}\right]$, but $\rho_{\mathrm{PBW}}\left(\left(P_{h}+P_{h^{\prime}}\right)^{2}\right)=$ $-\left[P_{h}, P_{h^{\prime}}\right]$. Therefore, $\rho_{\mathrm{PBW}} \neq \pi_{1}$. It also follows that $\operatorname{ker}^{\perp} \rho_{\mathrm{PBW}} \neq \operatorname{ker}^{\perp} \rho_{\perp}$, because for $h>h^{\prime} \in \mathrm{B}$, we have $\left(P_{h} P_{h^{\prime}} \mid\left[P_{h}, P_{h^{\prime}}\right]\right)=\left(P_{h} P_{h^{\prime}} \mid P_{h} P_{h^{\prime}}\right)-\left(P_{h} P_{h^{\prime}} \mid P_{h^{\prime}} P_{h}\right)>0$, since $P_{h} P_{h^{\prime}}, P_{h^{\prime}} P_{h}$ are linearly independent and have the same norm. Therefore, $\rho_{\mathrm{PBW}} \neq \rho_{\perp}$. Note that each choice of the basis in $\operatorname{Lie}(\mathrm{A})$ gives a different projection $\rho_{\mathrm{PBW}}$, so in this case, we actually define a class of examples.

The fourth one, a subclass of the previous one, is to take $\rho=\rho_{\text {Hall }}$, the projection derived as above taking a Hall basis in $\operatorname{Lie}(\mathrm{A})$. Recall that we described these basis in Section 2.4. In this case, Theorem 1 is equivalent to the following Gehrig-Kawski theorem.

Theorem 2 ([8] Theorem 28) Let $\mathcal{W}$ be a set of Hall words on the letters A. It follows that

$$
\sum_{w \in \mathrm{A}^{*}} w \otimes w=\exp \left(\sum_{h \in \mathcal{W}} \pi_{1}^{\prime}\left(\mathcal{S}_{h}\right) \otimes \mathcal{P}_{h}\right) \in \mathcal{A}
$$

In Tables 1 and 2, we gather known informations about $\operatorname{ker} \rho, \operatorname{ker}^{\perp} \rho=\operatorname{Lie}_{\rho}^{*}(\mathrm{~A})$ and basis in $\operatorname{Lie}(\mathrm{A})$ and its dual in $\operatorname{Lie}_{\rho}^{*}(\mathrm{~A})$ associated with projections $\rho$ just described. 
Table 1 Kernel and dual space associated with a projection $\rho$ on Lie(A)

\begin{tabular}{|c|c|c|c|}
\hline$\rho$ & $\operatorname{ker} \rho$ & & $\operatorname{ker}^{\perp} \rho=\operatorname{Lie}_{\rho}^{*}(\mathrm{~A})$ \\
\hline$\pi_{1}$ & $\left\{P^{k} \mid k \geq 2, P \in \operatorname{Lie}(\mathrm{A})\right\} \oplus \mathbb{K} 1$ & & $\operatorname{span}\left\{v ш w \mid v, w \in \mathrm{A}_{+}^{*}\right\}$ \\
\hline$\rho_{\perp}$ & $?$ & & $\operatorname{Lie}(A)$ \\
\hline$\rho_{\text {PBW }}$ & $\operatorname{span}\left\{\begin{array}{l}P_{h_{1}} \cdots P_{h_{k}} \mid \begin{array}{l}k \geq 2, \\
h_{1} \geq \cdots \geq h_{k} \in \mathrm{B}\end{array}\end{array}\right.$ & $\oplus \mathbb{K} 1$ & $\operatorname{span}\left\{S_{h} \mid h \in \mathrm{B}\right\}$ \\
\hline$\rho_{\text {Hall }}$ & $\operatorname{span}\left\{\mathcal{P}_{w} \mid w \in \mathrm{A}^{*} \backslash \mathcal{W}\right\}$ & & $\operatorname{span}\left\{\mathcal{S}_{h} \mid h \in \mathcal{W}\right\}$ \\
\hline
\end{tabular}

\section{Computing $\phi \circ \pi_{1}^{\prime}$}

Let $(R, \mu)$ be a $\mathbb{K}$-algebra with a multiplication $\mu: R \otimes R \rightarrow R$. If there is no confusion, we will use standard notation $\mathrm{ab}=\mu(a \otimes b)$. We also recursively introduce $\mu_{k}: R^{\otimes k} \rightarrow R$ by $\mu_{1}(a)=a, \mu_{2}=\mu, \mu_{k+1}=\mu \circ\left(\mu_{k} \otimes \mu_{1}\right)$. Let $\left(R^{\prime}, \cdot\right)$ be an $K$-algebra with a multiplication . : $R^{\prime} \times R^{\prime} \rightarrow R^{\prime}$ (we use simpler definition of this product, because we will not use its tensorial properties).

Let $\phi: \mathbb{K}_{w}\langle\langle\mathrm{~A}\rangle\rangle \rightarrow R$ and $\psi: \mathbb{K}\langle\mathrm{A}\rangle \rightarrow R^{\prime}$ be algebra homomorphisms. Then, it is easy to see that

$$
\phi \otimes \psi\left(\exp \left(\sum_{h \in \mathrm{B}} \pi_{1}^{\prime}\left(S_{h}\right) \otimes P_{h}\right)\right)=\exp \left(\sum_{h \in \mathrm{B}} \phi \circ \pi_{1}^{\prime}\left(S_{h}\right) \otimes \psi\left(P_{h}\right)\right) .
$$

From Theorem 1, we conclude that

$$
\phi \otimes \psi(\operatorname{Im}(\mathrm{Id}))=\exp \left(\sum_{h \in \mathrm{B}} \phi \circ \pi_{1}^{\prime}\left(S_{h}\right) \otimes \psi\left(P_{h}\right)\right) .
$$

Our aim is to give expression for $\phi \circ \pi_{1}^{\prime}$. For $k \in \mathbb{N}$, let us define a linear mapping $\phi_{k}: \mathbb{K}\langle\langle\mathrm{A}\rangle\rangle \rightarrow R$, for $v \in \mathrm{A}^{*}$ given by the following:

$$
\phi_{k}(v)=\sum_{v_{1} \cdots v_{k}=v} \phi\left(v_{1}\right) \cdots \phi\left(v_{k}\right),
$$

where the sum is taken over all $v_{i} \in \mathrm{A}^{*}$. We emphasize the existence of the empty word $1 \in \mathrm{A}^{*}$. In particular $\phi_{1}(v)=\phi(v)$.

Recall $I=\mathrm{Id}-\epsilon: \mathbb{K}\langle\mathrm{A}\rangle \rightarrow \mathbb{K}\langle\mathrm{A}\rangle$, and

$$
\pi_{1}^{\prime}=\sum_{k \geq 1} \frac{(-1)^{k+1}}{k} \operatorname{sh}_{k} \circ I^{\otimes k} \circ \delta_{k}^{\prime}
$$

Table 2 Basis in $\operatorname{Lie}(\mathrm{A})$ and its dual in $\operatorname{Lie}_{\rho}^{*}(\mathrm{~A})$ associated with a projection $\rho$ on $\operatorname{Lie}(\mathrm{A})$

\begin{tabular}{lll}
\hline$\rho$ & Basis in Lie(A) & Dual basis in $\operatorname{Lie}_{\rho}^{*}(\mathrm{~A})$ \\
\hline$\pi_{1}$ & $?$ & $\left\{\mathcal{S}_{h_{1}} w \cdots \amalg \mathcal{S}_{h_{k}} \mid k \geq 2, h_{1} \geq \cdots \geq h_{k} \in \mathcal{W}\right\}$ \\
$\rho_{\perp}$ & Any orthonormal one & The same one \\
$\rho_{\mathrm{PBW}}$ & $\left\{P_{h} \mid h \in \mathrm{B}\right\}$ & $\left\{S_{h} \mid h \in \mathrm{B}\right\}=?$ \\
$\rho_{\text {Hall }}$ & $\left\{\mathcal{P}_{h} \mid h \in \mathcal{W}\right\}$ & $\left\{\mathcal{S}_{h} \mid h \in \mathcal{W}\right\}$ \\
\hline
\end{tabular}


where $\operatorname{sh}_{k}$ and $\delta_{k}^{\prime}$ are defined by (2) and (3), respectively. Therefore,

$$
\phi \circ \pi_{1}^{\prime}=\sum_{k \geq 1} \frac{(-1)^{k+1}}{k} \mu_{k} \circ(\phi \circ I)^{\otimes k} \circ \delta_{k}^{\prime} .
$$

For $k \in \mathbb{N}$, let us define linear mappings $\tilde{\phi}_{k}: \mathbb{K}\langle\langle\mathrm{A}\rangle\rangle \rightarrow R$, for $v \in \mathrm{A}^{*}$ given by the following:

$$
\tilde{\phi}_{k}(v)=\sum_{u_{1} \cdots u_{k}=v} \phi\left(u_{1}\right) \cdots \phi\left(u_{k}\right)
$$

where the sum is taken over all $u_{i} \in \mathrm{A}_{+}^{*}$-the set of non-trivial words. Since $I$ annihilates empty word, it is easy to see that

$$
\mu_{k} \circ(\phi \circ I)^{\otimes k} \circ \delta_{k}^{\prime}(v)=\tilde{\phi}_{k}(v) .
$$

We conclude that

$$
\phi \circ \pi_{1}^{\prime}=\sum_{k \geq 1} \frac{(-1)^{k+1}}{k} \tilde{\phi}_{k}(v) .
$$

In the following lemma, we derive $\tilde{\phi}_{k}$ 's in terms of $\phi_{k}$ 's.

Lemma 4.1 It follows that

$$
\tilde{\phi}_{k}=\sum_{n=1}^{k}(-1)^{k-n}\left(\begin{array}{l}
k \\
n
\end{array}\right) \phi_{n}
$$

Proof Since in the definition of $\phi_{k}$ 's, we sum over all words and in the definition of $\tilde{\phi}_{k}$ 's, we sum over all non-empty words, we see that

$$
\phi_{k}=\tilde{\phi}_{k}+\left(\begin{array}{c}
k \\
1
\end{array}\right) \tilde{\phi}_{k-1}+\ldots+\left(\begin{array}{c}
k \\
k-1
\end{array}\right) \tilde{\phi}_{1} .
$$

Now, we use induction on $k$ to prove the hypothesis. For $k=1$, this is clear. Then, using (6) and then induction hypothesis, we have the following:

$$
\begin{aligned}
\tilde{\phi}_{k+1} & =\phi_{k+1}-\sum_{m=1}^{k}\left(\begin{array}{c}
k+1 \\
n
\end{array}\right) \tilde{\phi}_{k+1-m} \\
& =\phi_{k+1}-\sum_{m=1}^{k}\left(\begin{array}{c}
k+1 \\
n
\end{array}\right) \sum_{n=1}^{k+1-m}(-1)^{k+1-m-n}\left(\begin{array}{c}
k+1-m \\
n
\end{array}\right) \phi_{n} .
\end{aligned}
$$

Since $\left(\begin{array}{c}k+1 \\ m\end{array}\right)\left(\begin{array}{c}k+1-m \\ n\end{array}\right)=\left(\begin{array}{c}k+1 \\ n\end{array}\right)\left(\begin{array}{c}k+1-n \\ m\end{array}\right)$, and changing the order of summation, we have the following:

$$
\tilde{\phi}_{k+1}=\phi_{k+1}+\sum_{n=1}^{k}(-1)^{k-n}\left(\begin{array}{c}
k+1 \\
n
\end{array}\right)\left[\sum_{m=1}^{k+1-n}(-1)^{m}\left(\begin{array}{c}
k+1-n \\
m
\end{array}\right)\right] \phi_{n} .
$$

The expression in the square brackets equals -1 , and we are done.

Using this lemma, we see that

$$
\phi \circ \pi_{1}^{\prime}=\sum_{k \geq 1} \sum_{n=1}^{k} \frac{(-1)^{n+1}}{k}\left(\begin{array}{l}
k \\
n
\end{array}\right) \phi_{n}
$$


If $v_{m} \in \mathrm{A}_{m}^{*}$, then clearly $\tilde{\phi}_{n}\left(v_{m}\right)=0$ for all $n>m$ (since we can not divide $m$-letter word on $n$ non-trivial words). It means that

$$
\phi \circ \pi_{1}^{\prime}\left(v_{m}\right)=\sum_{k=1}^{m} \frac{(-1)^{k+1}}{k} \tilde{\phi}_{k}\left(v_{m}\right)=\sum_{k=1}^{m} \sum_{n=1}^{k} \frac{(-1)^{n+1}}{k}\left(\begin{array}{l}
k \\
n
\end{array}\right) \phi_{n}\left(v_{m}\right) .
$$

After changing the order of summation, we get

$$
\phi \circ \pi_{1}^{\prime}\left(v_{m}\right)=\sum_{n=1}^{m}(-1)^{n+1} \sum_{k=n}^{m} \frac{1}{k}\left(\begin{array}{l}
k \\
n
\end{array}\right) \phi_{n}\left(v_{m}\right) .
$$

A simple induction on $m$ shows that

$$
\sum_{k=n}^{m} \frac{1}{k}\left(\begin{array}{l}
k \\
n
\end{array}\right)=\frac{1}{n}\left(\begin{array}{c}
m \\
n
\end{array}\right),
$$

and therefore

$$
\phi \circ \pi_{1}^{\prime}\left(v_{m}\right)=\sum_{n=1}^{m} \frac{(-1)^{n+1}}{n}\left(\begin{array}{c}
m \\
n
\end{array}\right) \phi_{n}\left(v_{m}\right) .
$$

The above reasoning brings us to the following theorem.

Theorem 3 Let $S \in \mathbb{K}_{+}\langle\langle\mathrm{A}\rangle\rangle$, and assume $S=\sum_{m>1} S_{m}$, where $S_{m}$ is the homogeneous part spanned by words of length $m$, i.e., $S_{m}=\sum_{v \in \mathrm{A}_{m}^{*}}(S \mid v) v$. Then,

$$
\begin{aligned}
\phi \circ \pi_{1}^{\prime}(S) & =\sum_{m \geq 1} \sum_{n=1}^{m} \frac{(-1)^{n+1}}{n}\left(\begin{array}{c}
m \\
n
\end{array}\right) \phi_{n}\left(S_{m}\right) \\
& =\sum_{n=1}^{\infty} \sum_{m=n}^{\infty} \frac{(-1)^{n+1}}{n}\left(\begin{array}{c}
m \\
n
\end{array}\right) \phi_{n}\left(S_{m}\right) .
\end{aligned}
$$

\section{Signature of a Path}

Let $\gamma$ be a continuous path with finite variation in a finite dimensional linear space $V$ over $\mathbb{K}=\mathbb{R}$ equipped with the metric $d_{V}$. More precisely, take $T>0$ and let $t=\left(t_{1}, \ldots, t_{r}\right)$, such that $0=t_{0}<t_{1}<\cdots<t_{r} \leq T$ and $\# t:=r \in \mathbb{N}$. Denote the set off all such tuples by $\mathcal{P}$. For a continuous mapping $\gamma:[0, T] \rightarrow V$ its length is defined by the following:

$$
|\gamma|=\sup _{\mathbf{a} \in \mathcal{P}} \sum_{i=1}^{\# \mathbf{a}} d_{V}\left(\gamma_{t_{i}}, \gamma_{t_{i-1}}\right) .
$$

If $|\gamma|<+\infty$, then it is of finite variation. In particular, this implies that $\gamma$ is differentiable almost everywhere and

$$
\gamma_{t}=\int_{0}^{t} d \gamma_{\tau}
$$

for $t \in[0, T]$. Recursively define $X_{k}^{t}(\gamma) \in V^{\otimes k}$ for $k \in \mathbb{N}, t \in[0, T]$, by

$$
X_{1}^{t}(\gamma)=\int_{0}^{t} d \gamma_{\tau}, \quad X_{k+1}^{t}(\gamma)=\int_{0}^{t} X_{k}^{\tau}(\gamma) \otimes d \gamma_{\tau}
$$


Denote $X_{k}(\gamma)=X_{k}^{T}(\gamma)$ for $k>0$, and $X_{0}(\gamma)=1$ the neutral element in $T^{\otimes} V$. The signature of the path [13] $\gamma$ is

$$
X(\gamma)=1+X_{1}(\gamma)+X_{2}(\gamma)+X_{3}(\gamma)+\ldots=\sum_{k \geq 0} X_{k}(\gamma)
$$

in the tensor algebra $T^{\bar{\otimes}} V$.

In the space of continuous paths with finite variation, we introduce a natural concatenation product. Namely, for two paths $\gamma:[0, T] \rightarrow V, \tilde{\gamma}:[0, \tilde{T}] \rightarrow V$, we define the concatenation of these paths $\gamma \star \tilde{\gamma}:[0, T+\tilde{T}] \rightarrow V$ by

$$
\gamma \star \tilde{\gamma}_{t}=\left\{\begin{array}{ll}
\gamma(t) & t \in[0, T] \\
\tilde{\gamma}(t-T)-\tilde{\gamma}(0)+\gamma(T) & t \in[T, T+\tilde{T}]
\end{array} .\right.
$$

It follows from [2] that

$$
X(\gamma \star \tilde{\gamma})=X(\gamma) \otimes X(\tilde{\gamma}) .
$$

for any paths $\gamma, \tilde{\gamma}$. In particular, this means that for each $k \in \mathbb{N}$

$$
X_{k}(\gamma \star \tilde{\gamma})=X_{k}(\gamma)+X_{k-1}(\gamma) \otimes X_{1}(\tilde{\gamma})+\ldots+X_{1}(\gamma) \otimes X_{k-1}(\tilde{\gamma})+X_{k}(\tilde{\gamma})
$$

Clearly, this can be generalized to $X(\gamma \star \cdots \star \tilde{\gamma})=X(\gamma) \otimes \cdots \otimes X(\tilde{\gamma})$. We are particularly interested in a case $\gamma=\ldots=\tilde{\gamma}$, in which we use a notation $\gamma \star \cdots \star \gamma=\gamma^{\star k}$ for the $k$-times concatenation of $\gamma$.

Take a basis $\left(e_{i}\right)$ of $V$ and take an alphabet A consisting of the basis elements, i.e., $\mathrm{A}=\left\{e_{i}\right\}$. Define a natural algebra homomorphism $\iota_{\otimes}: \mathbb{K}\langle\mathrm{A}\rangle \rightarrow T^{\otimes} V$ given by $\iota_{\otimes}\left(e_{i_{1}} \cdots e_{i_{k}}\right)=e_{i_{1}} \otimes \cdots \otimes e_{i_{k}}$. Introduce a scalar product $(\cdot \mid \cdot): T^{\otimes} V \times T^{\otimes} V \rightarrow \mathbb{K}$ for which $\left\{\iota_{\otimes}(w) \mid w \in \mathrm{A}^{*}\right\}$ is an orthonormal basis. and define a linear homomorphism $\phi_{\gamma}: \mathbb{K}_{\Psi}\langle\langle\mathrm{A}\rangle\rangle \rightarrow \mathbb{K}$ given by $\phi_{\gamma}\left(e_{i_{1}} \cdots e_{i_{k}}\right)=\left(X(\gamma) \mid e_{i_{1}} \otimes \cdots \otimes e_{i_{k}}\right)$ and $\phi_{\gamma}(1)=1$.

Assuming $\gamma_{t}=\sum_{i} \gamma_{t}^{i} e_{i}$ we see that

$$
\phi_{\gamma}\left(e_{i_{1}} \cdots e_{i_{k}}\right)=\int_{0}^{T} \int_{0}^{\tau_{k}} \cdots \int_{0}^{\tau_{2}} d \gamma_{\tau_{1}}^{i_{1}} \cdots d \gamma_{\tau_{k}}^{i_{k}} .
$$

Chen [3] proved that $\phi_{\gamma}$ is a shuffle algebra homomorphism, i.e., $\phi_{\gamma}(v ш w)=\phi_{\gamma}(v) \phi_{\gamma}(w)$ for $v, w \in \mathrm{A}^{*}$. This means we can apply Theorem 2 in which we express $\phi_{\gamma} \circ \pi^{\prime}$ in terms of $\left(\phi_{\gamma}\right)_{k}$ (defined in (5)). Now observe that

$$
\begin{aligned}
\left(\phi_{\gamma}\right)_{k}(v) & =\sum_{v_{1} \cdots v_{k}=v} \phi_{\gamma}\left(v_{1}\right) \cdots \phi_{\gamma}\left(v_{k}\right) \\
& =\sum_{v_{1} \cdots v_{k}=v}\left(X(\gamma) \mid \iota_{\otimes}\left(v_{1}\right)\right) \cdots\left(X(\gamma) \mid \iota_{\otimes}\left(v_{k}\right)\right) \\
& =\left(X(\gamma) \otimes \cdots \otimes X(\gamma) \iota_{\otimes}(v)\right) \\
& =\left(X\left(\gamma^{\star k}\right) \mid \iota_{\otimes}(v)\right)=\phi_{\gamma^{\star k}}(v) .
\end{aligned}
$$

Finally, Theorem 3 states that

$$
\begin{aligned}
\phi_{\gamma} \circ \pi_{1}^{\prime}(S) & =\sum_{m \geq 1} \sum_{n=1}^{m} \frac{(-1)^{n+1}}{n}\left(\begin{array}{c}
m \\
n
\end{array}\right) \phi_{\gamma^{\star n}}\left(S_{m}\right) \\
& =\sum_{n=1}^{\infty} \sum_{m=n}^{\infty} \frac{(-1)^{n+1}}{n}\left(\begin{array}{c}
m \\
n
\end{array}\right) \phi_{\gamma^{\star n}}\left(S_{m}\right),
\end{aligned}
$$

where $S_{m}=\sum_{v \in \mathrm{A}_{m}^{*}}(S \mid v) v$. 
Let us also look what is the meaning of Theorem 2 in this context. Using canonical identification $\mathbb{K} \otimes T^{\bar{\otimes}} V \simeq T^{\bar{\otimes}} V$ and the definition of $\phi_{\gamma}$ we see that

$$
X(\gamma)=\phi_{\gamma} \otimes \iota_{\otimes}\left(\sum_{v \in \mathrm{A}^{*}} v \otimes v\right) .
$$

Since $\phi_{\gamma}$ is a shuffle algebra homomorphism, $\iota_{\otimes}$ a concatenation algebra homomorphism, and using Theorem 2, we conclude that

$$
\log X(\gamma)=\sum_{h \in \mathrm{B}} \phi_{\gamma} \circ \pi_{1}^{\prime}\left(S_{h}\right) \iota_{\otimes}\left(P_{h}\right)
$$

If for all $h \in \mathrm{B}, S_{h}$ is homogeneous of order $\# h$, i.e., $S_{h} \in \operatorname{span}\left\{w \in \mathrm{A}_{\# h}^{*}\right\}$ (this is the case for Hall bases), then

$$
\phi_{\gamma} \circ \pi_{1}^{\prime}\left(S_{h}\right)=\sum_{n=1}^{\# h} \frac{(-1)^{n+1}}{n}\left(\begin{array}{c}
\# h \\
n
\end{array}\right) \phi_{\gamma^{\star n}}\left(S_{h}\right) .
$$

This means that having got the signature of a path, the logarithm of this signature can be computed in terms of the signatures of concatenations of the path. For more information about log signature (see $[6,15,19])$.

Open Access This article is distributed under the terms of the Creative Commons Attribution 4.0 International License (http://creativecommons.org/licenses/by/4.0/), which permits unrestricted use, distribution, and reproduction in any medium, provided you give appropriate credit to the original author(s) and the source, provide a link to the Creative Commons license, and indicate if changes were made.

\section{References}

1. Chapoton F, Patras F. Enveloping algebras of preLie algebras, Solomon idempotents and the Magnus formula. International Journal of Algebra and Computation. 2013;23(04):853-61.

2. Chen K-T. Iterated integrals and exponential homomorphisms. Proc London Math Soc. 1954;4(3): 502-12.

3. Chen K-T. Algebraic paths. J Algebra. 1968;10:8-36.

4. Chow W-L. ÜBer Systeme von linearen partiellen Differentialgleichungen erster Ordnung. Math Ann. 1939;117(1):98-105.

5. Duchamp G. Orthogonal projection onto the free Lie algebra. Theor Comput Sci. 1991;79(1):227-39.

6. Friz PK, Victoir NB. Multidimensional stochastic processes as rough paths: theory and applications. Cambridge: Cambridge University Press; 2010.

7. Gehrig E, Kawski M. A Hopf-algebraic formula for compositions of noncommuting flows. In: 47th IEEE conference on decision and control, 2008. CDC 2008, IEEE; 2008. p. 1569-74.

8. Gehrig ET. Hopf algebras, projections, and coordinates of the first kind in control theory. Arizona: Arizona State University; 2007.

9. Hall M. A basis for free Lie rings and higher commutators in free groups. Proc Am Math Soc. 1950;1(5):575-81.

10. Kawski M. Bases for Lie algebras and a continuous $\mathrm{CBH}$ formula. Unsolved problems in mathematical systems and control theory. In: Blondel VD and Megretski A, editors. Princeton: Princeton University Press; 2004.

11. Kawski M, Sussmann HJ. Noncommutative power series and formal Lie-algebraic techniques in nonlinear control theory. In: Operators, systems, and linear algebra (Kaiserslautern, 1997), European Consort. Math. Indust. Teubner, Stuttgart; 1997. p. 111-28.

12. Lyons T, Qian Z. System control and rough paths. Oxford: Oxford University Press; 2002.

13. Lyons TJ. Differential equations driven by rough signals. Revista Matemática Iberoamericana. 1998;14(2):215-310.

14. Lyons TJ, Caruana M, Lévy T. Differential equations driven by rough paths. Berlin: Springer; 2007. 
15. Lyons TJ, Sidorova N, et al. On the radius of convergence of the logarithmic signature. Ill J Math 50. 2006;1-4:763-90.

16. Melançon G, Reutenauer C. Lyndon words, algebras, free and shuffles. Canad J Math. 1989;41(4): 577-91.

17. Radford DE. A natural ring basis for the shuffle algebra and an application to group schemes. Journal of Algebra. 1979;58(2):432-54.

18. Rashevskii PK. About connecting two points of complete non-holonomic space by admissible curve (in Russian). Uch Zapiski ped inst Libknexta. 1938;2:83-94.

19. Reizenstein J, Graham B. The iisignature library: efficient calculation of iterated-integral signatures and log signatures. arXiv:1802.08252. 2018.

20. Reutenauer C. Free Lie algebras, vol. 7 of London mathematical society monographs. New Series. New York: The Clarendon Press Oxford University Press; 1993. Oxford Science Publications.

21. Shirshov A. On the bases of a free Lie algebra. Algebra Logika. 1962;1(1):14-9.

22. Sussmann HJ. A product expansion for the Chen series. In: Theory and applications of nonlinear control systems (Stockholm, 1985), North-Holland, Amsterdam; 1986. p. 323-35.

23. Viennot G. Algèbres de Lie libres et monoïdes libres, vol. 691 of Lecture Notes in Mathematics. Berlin: Springer; 1978. Bases des algèbres de Lie libres et factorisations des monoïdes libres. 